\title{
Primary small cell neuroendocrine carcinoma of the Bartholin's gland: A case report
}

\author{
JIANG-CHUN WU $^{1 *}$, MEI-LI XI ${ }^{1 *}$, YAN-QIU WANG ${ }^{2}$, WEN-BIN TANG $^{1}$ and YU-QIN ZHANG ${ }^{1}$ \\ Departments of ${ }^{1}$ Obstetrics and Gynecology, and ${ }^{2}$ Pathology, Zhongshan Hospital, \\ Fudan University, Shanghai 200032, P.R. China
}

Received November 7, 2017; Accepted April 23, 2018

DOI: $10.3892 / \mathrm{ol} .2018 .9231$

\begin{abstract}
Primary carcinoma of the Bartholin's gland (BG) is a rare malignancy. There are extremely rare cases of small cell neuroendocrine carcinoma (SCNC) of the BG reported in the English literature. A postmenopausal female presented with a 1-month history of increasing pain and swelling on the left vulva consistent with spontaneously bleeding. Pathology identified SCNC that arose in BG. The patient was treated with a radical wide local excision and bilateral inguinal lymph node dissection followed by six courses of chemotherapy. One month after primary treatment, without any pelvic recurrence or abnormal tumor markers indications, distant metastasis of the liver was diagnosed and VI hepatic lobectomy was performed. The patient maintained regular adjuvant chemotherapy every month under outpatient surveillance and has no local recurrence or distant metastasis.
\end{abstract}

\section{Introduction}

Primary carcinoma of the Bartholin's gland (BGC) is an extremely rare malignancy accounting for $<5 \%$ of all vulvar malignancies and representing $0.001 \%$ of gynecological cancers in the United States (1-3). Adenocarcinoma (ACC) and squamous cell carcinoma (SCC) are the predominating types of carcinoma at this site, occurring alongside $\sim 80 \%$ of cases of BGC (equal frequency between ACC and SCC) (4). Di Donato et al (2017) conducted a literature search for all manuscripts discussing BGC, and identified only 3 reported cases of small cell neuroendocrine carcinoma (SCNC) in the Bartholin's gland (BG) (5-7). SCNC progresses aggressively,

Correspondence to: Dr Yu-Qin Zhang, Department of Obstetrics and Gynecology, Zhongshan Hospital, Fudan University, 180 Fenglin Road, Shanghai 200032, P.R. China

E-mail: zhangyuqin201710@163.com

"Contributed equally

Key words: small cell neuroendocrine carcinoma, radical wide local excision, bilateral inguinal lymph node dissection, chemotherapy, metastasis of the liver producing early local recurrences and distant metastases, resulting in eventual patient mortality. The present case report details a case of well-characterized SCNC that arose in BG associated with a later hepatic metastasis following 6 months' chemotherapy treatment with no local recurrence or distant metastasis. Continued studies of diagnosis and treatment are required in order to improve management of this rare malignancy.

\section{Case report}

A 56-year-old postmenopausal female presented with a 1-month history of increasing pain and swelling on the left vulva with consistent bleeding. The patient first sought medical attention at Shanghai Feng'ai Hospital in September 2016. A biopsy demonstrated an ACC that arouse in BG. The maximum diameter of the tumor was $\sim 30 \mathrm{~mm}$. The patient sought additional treatment at Zhongshan Hospital of Fudan University (Shanghai, China) in December 2016. Gynecological examination revealed a $30 \mathrm{~mm}$ ulcerated, indurated lump involving the left labium majus. No inguinal or supraclavicular nodes were palpable. Magnetic resonance imaging of the pelvis identified a $30 \mathrm{~mm}$ solid mass with intermediate signal on T1-weighted imaging and a slightly high signal on T2-weighted imaging arising from the left BG (Fig. 1). A metastatic workup computed tomography scan, which included examination of the whole torso, did not demonstrate any metastatic disease. The patient's serum level of neuron specific enolase (NSE) was also within the normal range (normal level $\sim 15.2 \mathrm{ng} / \mathrm{ml}$ ). The biopsy specimen was reanalyzed by a pathologist of Zhongshan Hospital of Fudan University and a SCNC that arose in BG was identified (Fig. 2). The patient was surgically treated with a wide local excision and a bilateral inguinal lymph node dissection. An intraoperative frozen section was sent to the laboratory to confirm the free resection margin. Final pathology confirmed the diagnosis of a SCNC of BG with free surgical margin (Fig. 3). No inguinal lymph nodes were positive for metastatic tumor growth (Fig. 4). Following surgery, six courses of adjuvant chemotherapy (cisplatin $2 \mathrm{mg} / \mathrm{kg}$ and etoposide $5 \mathrm{mg} / \mathrm{kg}$, every 21 days) were performed. After 1 month, the patient maintained regular outpatient surveillance. Unfortunately, distant metastasis was identified in June 2017. Magnetic Resonance Cholangiopancreatography identified an 
$18 \mathrm{~mm}$ ovoid shape with low signal on T1-weighted imaging and high signal on T2-weighted imaging in the right hepatic lobe (Fig. 5). Positron emission tomography scanning detected fluorodeoxyglucose-avid lesions in the right hepatic lobe and did not demonstrate any additional distant disease including the local vulva (Fig. 6). The serum NSE and $\alpha$-fetoprotein (AFP) were also in the normal range. A VI hepatic lobectomy was performed. Postoperative pathology identified a SCNC that arose in BG with hepatic metastasis (Fig. 7). The patient was administered regular adjuvant chemotherapy (using the treatment regimen described above) every month under outpatient surveillance. The patient provided written informed consent for the publication of their data.

Immunohistochemical procedures were performed as follows: Tissue was fixed with $10 \%$ buffered formalin (Thermo Fisher Scientific, Inc., Waltham, MA, USA) for $30 \mathrm{~min}$ at room temperature, then embedded in paraffin wax for $3 \mathrm{~h}$ at $60^{\circ} \mathrm{C}$ and cut into $5 \mu \mathrm{m}$ thick sections. Dewaxing was performed with $5 \%$ xylene (Thermo Fisher Scientific, Inc.) for $20 \mathrm{~min}$ twice at room temperature, and tissue sections were rehydrated through a graded series of ethanol solutions (100, 95, 90 and 80\% each for 5 min; Thermo Fisher Scientific, USA). Endogenous peroxidase activity was blocked by incubating slides in $1 \% \mathrm{H}_{2} \mathrm{O}_{2}$ (Thermo Fisher Scientific, Inc.) in methanol for 15-20 min at room temperature. Slides were washed with running deionized water for $2 \mathrm{~min}$. Non-specific binding was blocked by incubating sections in $2.5 \%$ fetal bovine serum (Hyclone; GE Healthcare Life Sciences, Logan, UT, USA) diluted in Tris-buffered saline (TBS; Sigma-Aldrich; Merck KGaA, Darmstadt, Germany) with $0.1 \%$ bovine serum albumin (BSA; Sigma-Aldrich; Merck KGaA) for $30 \mathrm{~min}$ at room temperature. Excess serum was removed without washing and replaced with 100-300 $\mu 1$ mouse monoclonal anti-SYN (1:100; cat. no. 4329), mouse monoclonal CAM 5.2 (1:200; cat. no. 3362S), rat monoclonal CD20 (1:200; cat. no. 23543) or rat monoclonal anti-CHG (1:100 cat. no. 85339; all Cell Signaling Technology, Inc., Danvers, MA, USA) diluted in TBS with $0.1 \%$ BSA. Samples were incubated at room temperature for $60 \mathrm{~min}$ in a humid chamber. Subsequent secondary antibodies, including Cy3-conjugated goat anti-mouse, or anti-rat which were diluted in TBS with $0.1 \%$ BSA (anti-mouse IgG, 1:300, cat. no. 4410; anti-rat $\operatorname{IgG}, 1: 400$ or 1:500, cat. no. 4417; Cell Signaling Technology, Inc.) was placed on the sections and incubated for $30 \mathrm{~min}$ at room temperature, then washed off with PBS (Hyclone; GE Healthcare Life Sciences) for $5 \mathrm{~min}$ twice and exposed to streptavidin-peroxidase conjugate (Vector Laboratories, Ltd., Peterborough, UK) for $30 \mathrm{~min}$ at room temperature. The bound antibody-peroxidase complexes on the sections were visualized using a 3,3-diaminobenzidine tetrahydrochloride (DAB; Cell Signaling Technology) substrate solution consisting of $1.5 \mathrm{mg} \mathrm{DAB}$ and $50 \mu \mathrm{l}$ of $30 \%$ hydrogen peroxide in $10 \mathrm{ml}$ of $0.1 \mathrm{M}$ Tris- $\mathrm{HCl}$ (both Thermo Fisher Scientific, Inc.), pH 7.6. The sections were incubated in the dark until brown staining appeared for $12 \mathrm{~h}$ at $4^{\circ} \mathrm{C}$, washed in PBS, counterstained with hematoxylin, dehydrated, and mounted with Permount (both Thermo Fisher Scientific, Inc.). A low-power view of the tumor biopsy was obtained using a light microscope (magnification, x20; Thermo Fisher Scientific, Inc.).

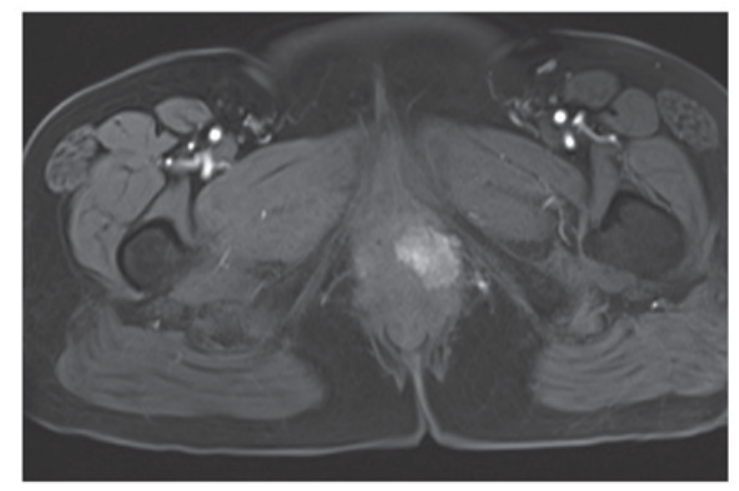

Figure 1. Magnetic resonance imaging of the pelvis reveals a $3.3 \times 2.6 \mathrm{~cm}$ solid mass with intermediate signal on T1-weighted imaging arising from the left Bartholin's gland.

\section{Discussion}

$\mathrm{BGC}$ is a rare malignancy and accounts for fewer than $5 \%$ of all vulvar carcinomas (7). SCNC arising in BG is extremely rare, and only 3 cases have been reported in English literature (3). A foreign language literature search was not performed. Due to the potentially aggressive behavior of SCNC, prompt diagnosis is required (8). However, SCNC of the BG is often diagnosed late since the lesions are deep within the vulva and present with similar symptoms to most vulvar diseases, including abscesses or cysts (9). Patients usually complain of pain, swelling on the vulva, dyspareunia and bleeding $(10,11)$. In cases where BGC is suspected, the clinical diagnostic criteria is as follows: The tumor must be primarily located in the BG area; the surrounding skin must be undamaged; areas of apparent transition from normal to neoplastic elements must be observed; the histological tumor type must be consistent with the BG origin; there must be no evidence of a previous or subsequent primary tumor of similar histologic type elsewhere $(12,13)$. Di Donato et al (7) collected all published manuscripts regarding $\mathrm{BGC}$ and the median age of patients was $52.99 \pm 13.94$ years. Therefore, independent of whether a patient is pre- or post-menopausal, every mass within the BG area should be considered as a potential carcinoma until proven to be benign with biopsies of adequate size and depth $(4,14)$. Diagnosis of BGC is established based upon histological examination. Abundant apoptotic debris and mitotic figures may be seen in SCNC of BG, and immunohistochemical stains were strongly positive for CAM 5.2, NSE, SYN, CHG, CD56 and CD10 (7,15-17). In the present case report, immunohistochemical stains obtained by the Shanghai Feng'ai Hospital were strongly positive for CAM 5.2 and CD20 and demonstrated an ACC that arose in BG. The biopsy was reanalyzed by pathologists of Zhongshan Hospital of Fudan University, and the postoperative pathology supported the diagnosis of SCNC via the positive presence of SYN and CHG.

There is presently no consensus on the treatment of BGC due to the lack of randomized controlled trials and large cases in the literature. The treatment of BGC may include extensive vulvar surgery, and inguinal and pelvic lymphadenectomy, similar to treatment of SCC of the vulva. SCNC is a subtype of neuroendocrine cancer, resembling small cell carcinoma of the lung (18). It has a poor prognosis 


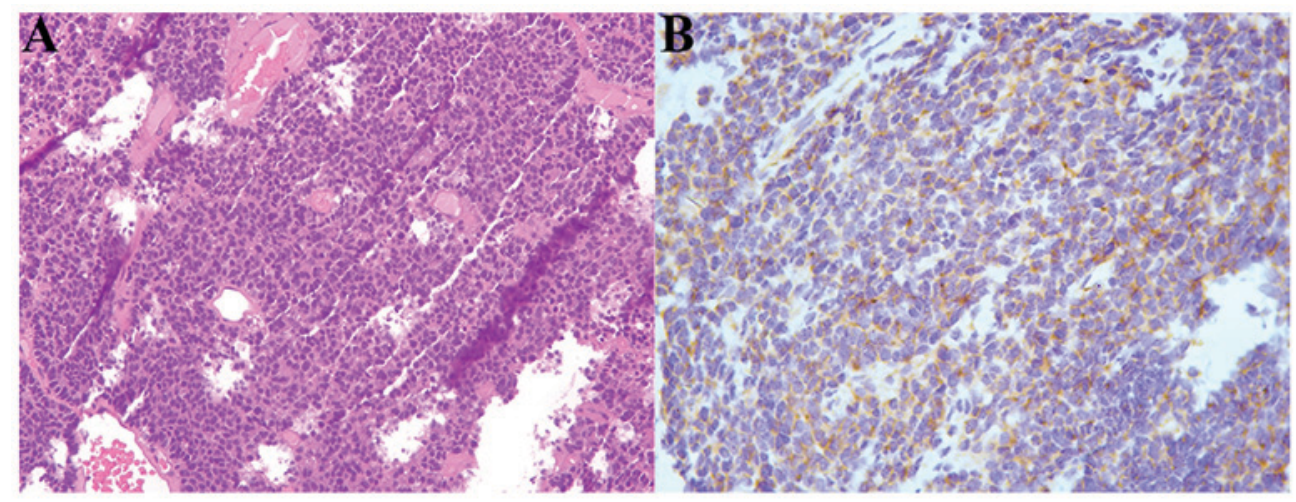

Figure 2. A low-power view of the tumor biopsy under a light microscope. (A) Hematoxylin and eosin staining. (B) Chromogranin staining. In total, $50 \%$ of the tumor cells are positive (magnification, $\mathrm{x} 20$ ).

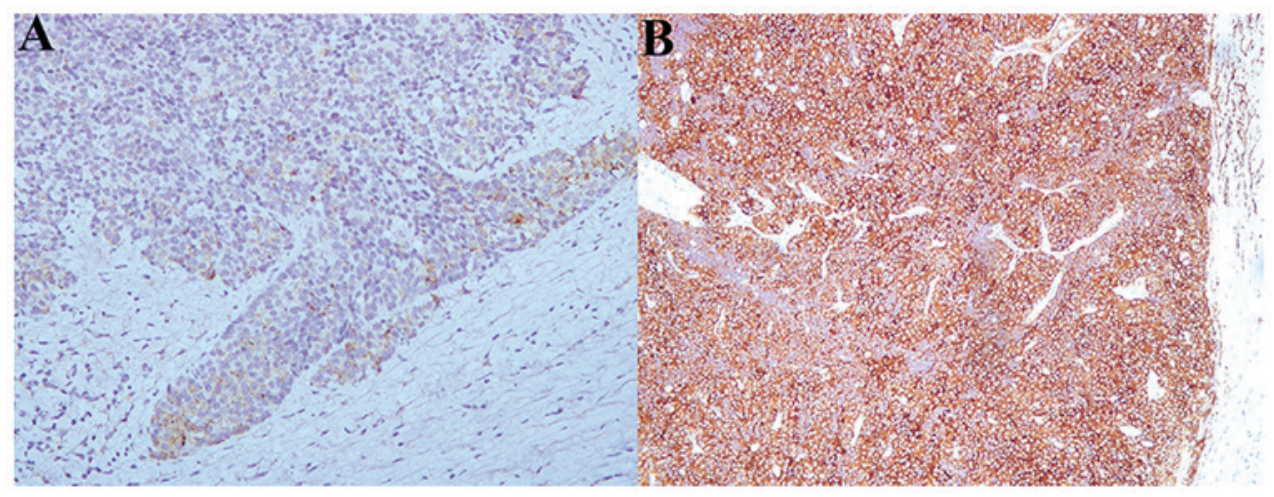

Figure 3. Low-power view of the tumor sample from vulvar postoperative pathology. (A) Chromogranin staining. (B) Synaptophysin staining (magnification, x20).

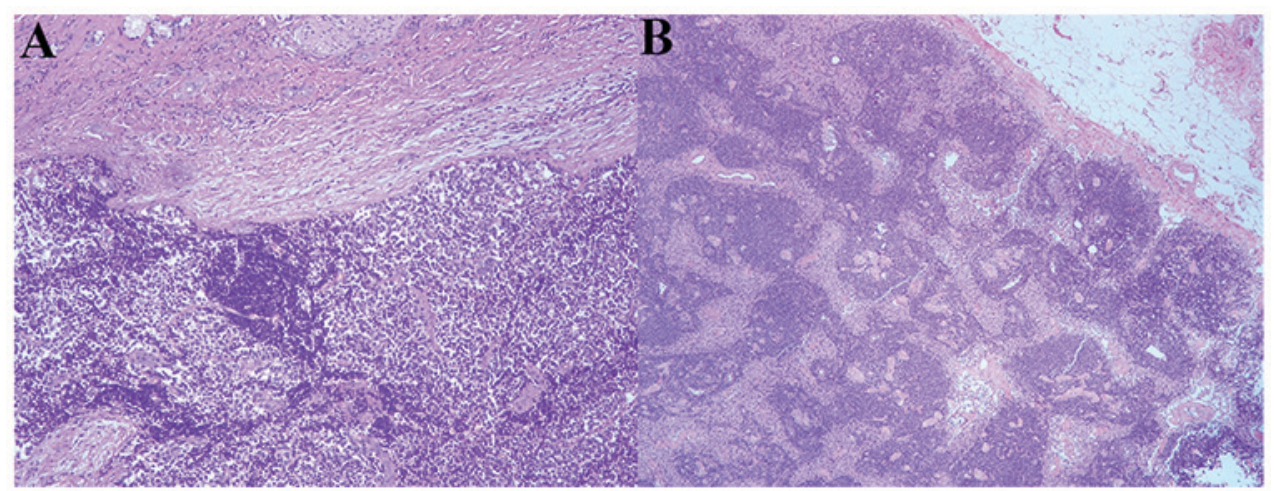

Figure 4. Low-power view of the inguinal lymph nodes. The (A) right and (B) left nodes (magnification, x20).

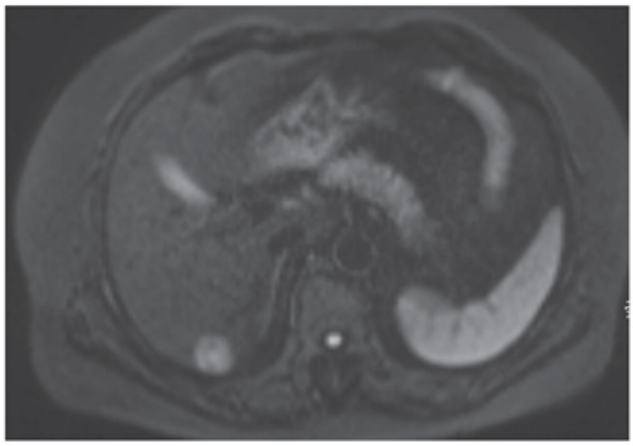

Figure 5. Magnetic Resonance Cholangiopancreatography identified an $18 \mathrm{~mm}$ ovoid shape with low signal on T1-weighted imaging. and the use of cisplatin and etoposide is recommended as in small cell pulmonary tumors (19-21). In the present case, one month after primary treatment, distant metastasis of the liver was diagnosed, however the patient did not exhibit any other distance disease, including disease of the local vulva. Furthermore, the serum NSE and AFP levels, (normal $\sim 20 \mathrm{ng} / \mathrm{ml}$ ) were also in the normal range. As a result of the distant metastasis, regular outpatient surveillance was not limited to the pelvic cavity and tumor markers; general examinations were also carried out. When the metastasis of the liver was diagnosed, a VI hepatic lobectomy was performed and primary chemotherapy was performed every 21 days (cisplatin $2 \mathrm{mg} / \mathrm{kg}$ and etoposide $5 \mathrm{mg} / \mathrm{kg}$, daily). 


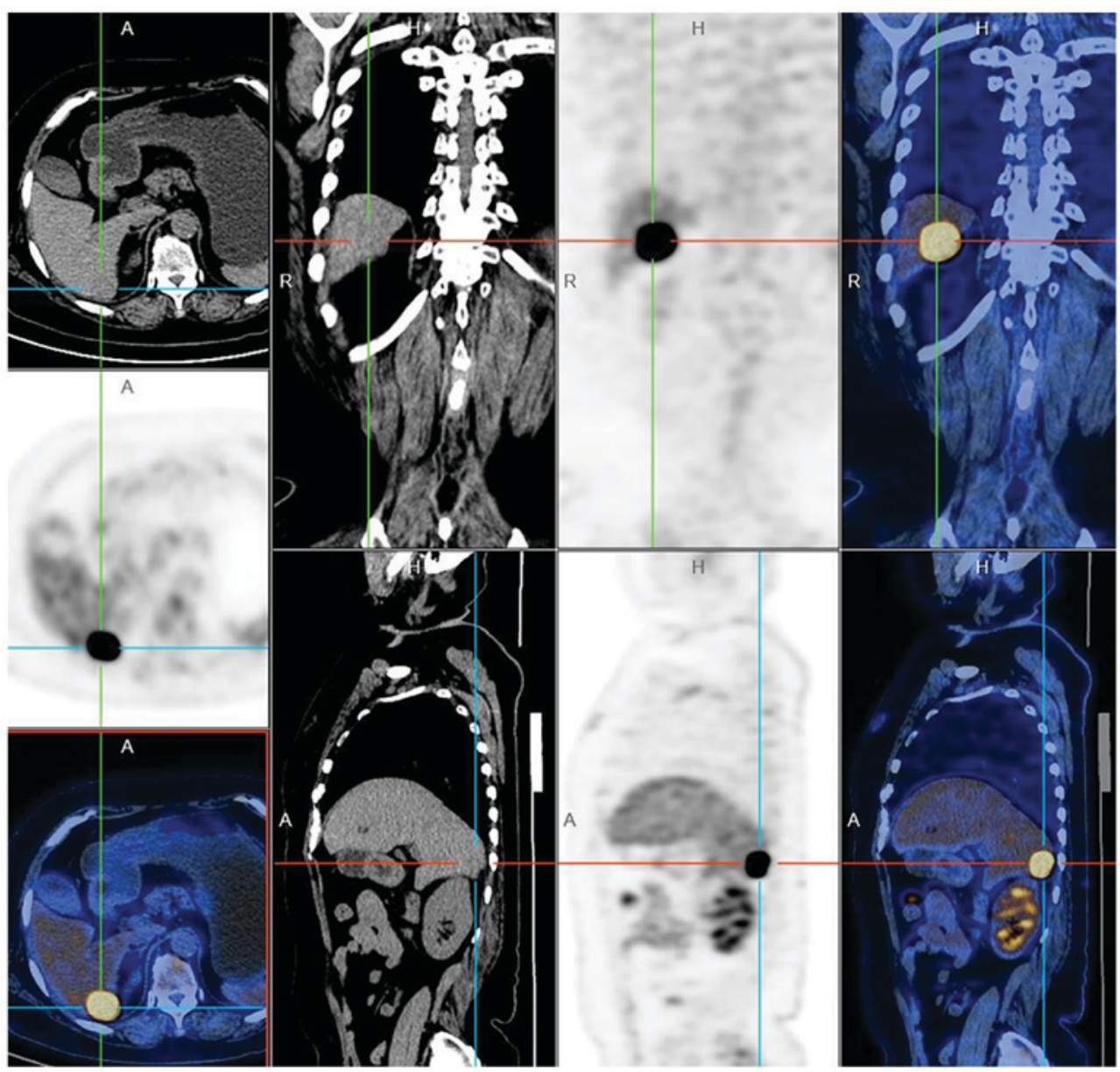

Figure 6. 18-Fluorodeoxyglucose positron emission tomography scanning did not demonstrate any other distant metastasis.

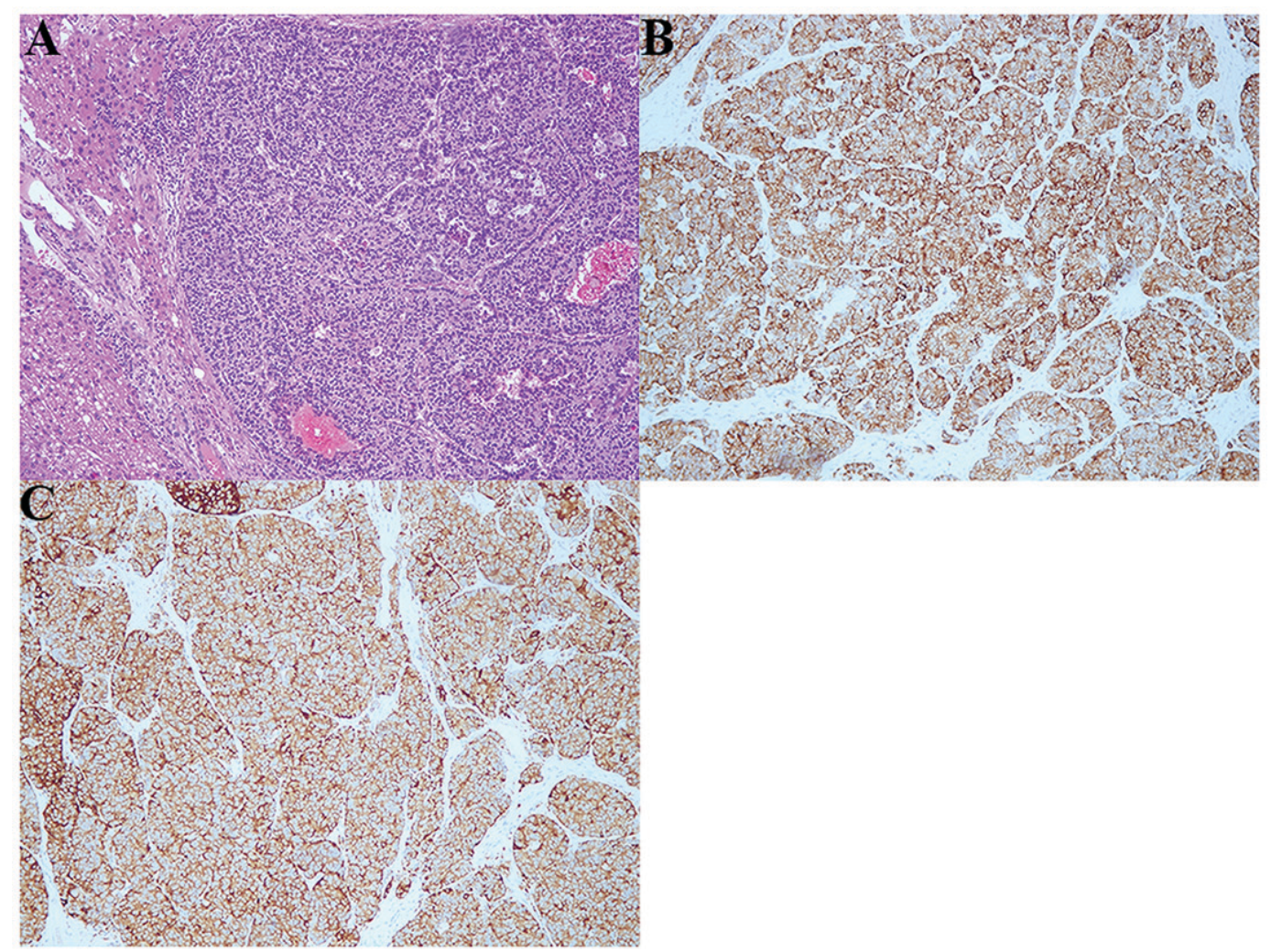

Figure 7. Low-power view of the tumor of hepatic metastasis. (A) Hematoxylin and eosin staining. (B) Chromogranin staining. (C) Synaptophysin staining (magnification, $\mathrm{x} 20$ ). 
The treatment strategy remained unchanged since SCNC of $\mathrm{BG}$ is rare, thus there was no definitive treatment guideline available to follow. As a result, physicians utilized the pulmonary strategy of adjuvant chemotherapy (cisplatin and etoposide) (21). Additionally, the lesions of the liver were completely excised, meaning the patient had no local recurrence or distant metastasis, confirmed via regular outpatient surveillance.

To conclude, primary BGC is a rare form of vulvar cancer. BGC remains a challenge for gynecologic oncologists to treat. The initial diagnosis is often delayed because of the absence of specific symptoms and its potential for misdiagnosis as a benign disease. A case of early stage SCNC arising from BG is reported based on morphological and immunohistochemical criteria. A delay in the diagnosis is not uncommon but prompt recognition may aid treatment. The present case underscores a potential need for biopsy and excision of BG cysts when they present, in order to screen for the clinical diagnostic criteria of BGC. Additional education for patients and primary providers is required in order to avoid misdiagnosis and improve early diagnosis of BGC. Due to the rarity of SCNC, current therapeutic guidelines have not been standardized. Presently, the treatment modalities used are similar to other forms of vulvar carcinoma and the outcomes appear to be similar and do not have good recurrence-free survival or overall survival rates. Therefore, it is important to report additional cases of BGC and conduct clinical trials to obtain a clinical consensus on treatment.

\section{Acknowledgements}

The present study was supported by Zhongshan Hospital of Fudan University.

\section{Funding}

No funding was received.

\section{Availability of data and materials}

All data generated or analyzed during this study are included in this published article.

\section{Authors' contributions}

Conception and design: YQZ, JCW, MLX and WBT. Collection and assembly of data: MLX and YQW. Data analysis and interpretation: YQZ, JCW, MLX, WBT and YQW. Manuscript writing: YQZ, JCW, MLX, WBT and YQW. Final approval of manuscript: YQZ, JCW, MLX, WBT and YQW.

\section{Ethics approval and consent to participate}

Not applicable.

\section{Patient consent for publication}

The patient provided written informed consent for the publication of their data.

\section{Competing interests}

The authors declare that they have no competing interest.

\section{References}

1. DePasquale SE, McGuinness TB, Mangan CE, Husson M and Woodland MB: Adenoid cystic carcinoma of Bartholin's gland: A review of the literature and report of a patient. Gynecol Oncol 61: 122-125, 1996.

2. Chamlian DL and Taylor HB: Primary carcinoma of Bartholin's gland. A report of 24 patients. Obstet Gynecol 39: 489-494, 1972.

3. Nasu K, Kawano Y, Takai N, Kashima K and Miyakawa I: Adenoid cystic carcinoma of Bartholin's gland. Case report with review of the literature. Gynecol Obstet Invest 59: 54-58, 2005.

4. Ouldamer L, Chraibi Z, Arbion F, Barillot I and Body G: Bartholin's gland carcinoma: Epidemiology and therapeutic management. Surg Oncol 22: 117-122, 2013.

5. Obermair A, Koller S, Crandon AJ, Perrin L and Nicklin JL: Primary Bartholin gland carcinoma: A report of seven cases. Aust N Z J Obstet Gynaecol 41: 78-81, 2001.

6. Jones MA, Mann EW, Caldwell CL, Tarraza HM, Dickersin GR and Young RH: Small cell neuroendocrine carcinoma of Bartholin's gland. Am J Clin Pathol 94: 439-442, 1990.

7. Di Donato V, Casorelli A, Bardhi E, Vena F, Marchetti C, Muzii L and Benedetti Panici P: Bartholin gland cancer. Crit Rev Oncol Hematol 117: 1-11, 2017.

8. Khoury-Collado F, Elliott KS, Lee YC, Chen PC and Abulafia O: Merkel cell carcinoma of the Bartholin's gland. Gynecol Oncol 97: 928-931, 2005.

9. Bhalwal AB, Nick AM, Dos Reis R, Chen CL, Munsell MF Ramalingam P, Salcedo MP, Ramirez PT, Sood AK and Schmeler KM: Carcinoma of the Bartholin gland: A Review of 33 cases. Int J Gynecol Cancer 26: 785-789, 2016.

10. Yang SY, Lee JW, Kim WS, Jung KL, Lee SJ, Lee JH, Bae DS and Kim BG: Adenoid cystic carcinoma of the Bartholin's gland: Report of two cases and review of the literature. Gynecol Oncol 100: 422-425, 2006.

11. Frable WJ and Goplerud DR: Adenoid cystic carcinoma of Bartholin's gland diagnosis by aspiration biopsy. Acta Cytol 19: 152-153, 1975.

12. Copeland LJ, Sneige N, Gershenson DM, Saul PB, Stringer CA and Seski JC: Adenoid cystic carcinoma of Bartholin gland. Obstet Gynecol 67: 115-120, 1986.

13. Lee MY, Dalpiaz A, Schwamb R, Miao Y, Waltzer W and Khan A: Clinical pathology of Bartholin's glands: A Review of the literature. Curr Urol 8: 22-25, 2015.

14. Rosén C and Malmström H: Invasive cancer of the vulva. Gynecol Oncol 65: 213-217, 1997.

15. Groben P, Reddick R and Askin F: The pathologic spectrum of small cell carcinoma of the cervix. Int J Gynecol Pathol 4: 42-57, 1985.

16. Fried-Oginski W, Lovecchio JL, Farahani G and Smilari T: Malignant myxoid sarcoma of the Bartholin gland in pregnancy. Am J Obstet Gynecol 173: 1633-1635, 1995.

17. González-Bugatto F, Añón-Requena MJ, López-Guerrero MA, Báez-Perea JM, Bartha JL and Hervías-Vivancos B: Vulvar leiomyosarcoma in Bartholin's gland area: A case report and literature review. Arch Gynecol Obstet 279: 171-174, 2009.

18. Dakhil CS, Wick JA, Kumar AK, Satyan MT and Neupane P: Extrapulmonary small cell carcinoma: The University of Kansas experience and review of literature. Med Oncol 31: 187, 2014.

19. Yildirim Y, Elagoz S, Koyuncu A, Aydin C and Karadayi K: Management of neuroendocrine carcinomas of the breast: A rare entity. Oncol Lett 2: 887-890, 2011.

20. Watrowski R, Jäger C, Mattern D and Horst C: Neuroendocrine carcinoma of the breast-diagnostic and clinical implications. Anticancer Res 32: 5079-5082, 2012.

21. Ramirez RA, Chauhan A, Gimenez J, Thomas KEH, Kokodis I and Voros BA: Management of pulmonary neuroendocrine tumors. Rev Endocr Metab Disord 18: 433-442, 2017.

This work is licensed under a Creative Commons Attribution-NonCommercial-NoDerivatives 4.0 International (CC BY-NC-ND 4.0) License. 